\title{
Spatial Complexity Measure for Characterising Cellular Automata Generated 2D Patterns
}

\author{
Mohammad Ali Javaheri Javid, Tim Blackwell, Robert Zimmer, Mohammad \\ Majid al-Rifaie \\ Department of Computing \\ Goldsmiths, University of London \\ London SE14 6NW, UK \\ $\{$ m.javaheri,t.blackwell,r.zimmer,m.majid\}@gold.ac.uk
}

Keywords: cellular automata, spatial complexity, 2D patterns

\begin{abstract}
Cellular automata (CA) are known for their capacity to generate complex patterns through the local interaction of rules. Often the generated patterns, especially with multi-state two-dimensional CA, can exhibit interesting emergent behaviour. This paper addresses quantitative evaluation of spatial characteristics of CA generated patterns. It is suggested that the structural characteristics of two-dimensional (2D) CA patterns can be measured using mean information gain. This information-theoretic quantity, also known as conditional entropy, takes into account conditional and joint probabilities of cell states in a $2 \mathrm{D}$ plane. The effectiveness of the measure is shown in a series of experiments for multi-state 2D patterns generated by CA. The results of the experiments show that the measure is capable of distinguishing the structural characteristics including symmetry and randomness of 2D CA patterns.
\end{abstract}

\section{Introduction}

Cellular automata (CA) are one of the early bio-inspired systems invented by von Neumann and Ulam in the late 1940s to study the logic of self-reproduction in a material-independent framework. CA are known to exhibit complex behaviour from the iterative application of simple rules. The popularity of the Game of Life drew the attention of a wider community of researchers to the unexplored potential of CA applications and especially in their capacity to generate complex behaviour. The formation of complex patterns from simple rules sometimes with high aesthetic qualities has been contributed to the creation of many digital art works since the 1960s. The most notable works are "Pixillation", one of the early computer generated animations [11], the digital art works of Struycken [10], Brown [3] and evolutionary architecture of Frazer [5]. Furthermore, CA have been used for music composition, for example, Xenakis [17] and Miranda [9].

Although classical one-dimensional CA with binary states can exhibit complex behaviours, experiments with multi-state two-dimensional (2D) CA reveal a very rich spectrum of symmetric and asymmetric patterns $[6,7]$. 
There are numerous studies on the quantitative [8] and qualitative behaviour [14-16] of CA but they are mostly concerned with categorising the rule space and the computational properties of CA. In this paper, we investigate information gain as a spatial complexity measure of multi-state 2D CA patterns. Although the Shannon entropy is commonly used to measure complexity, it fails to discriminate accurately structurally different patterns in two-dimensions. The main aim of this paper is to demonstrate the effectiveness of information gain as a measure of $2 \mathrm{D}$ structural complexity.

This paper is organised as follows. Section 2 provides formal definitions and establishes notation. Section 3 demonstrates that Shannon entropy is an inadequate measure of $2 \mathrm{D}$ cellular patterns. In the framework of the objectives of this study a spatial complexity spectrum is formulated and the potential of information gain as a structural complexity measure is discussed. Section 4 gives details of experiments that test the effectiveness of information gain. The paper closes with a discussion and summary of findings.

\section{Cellular Automata}

This section serves to specify the cellular automata considered in this paper, and to define notation.

A cellular automaton $\mathcal{A}$ is specified by a quadruple $\langle L, S, N, f\rangle$ where:

- $L$ is a finite square lattice of cells $(i, j)$.

$-S=\{1,2, \ldots, k\}$ is set of states. Each cell $(i, j)$ in $L$ has a state $s \in S$.

$-\mathcal{N}$ is neighbourhood, as specified by a set of lattice vectors $\left\{e_{a}\right\}, a=$ $1,2, \ldots, N$. The neighbourhood of cell $r=(i, j)$ is $\left\{r+e_{1}, r+e_{2}, \ldots, r+e_{N}\right\}$. A cell is considered to be in its own neighbourhood so that one of $\left\{e_{a}\right\}$ is the zero vector $(0,0)$. With an economy of notation, the cells in the neighbourhood of $(i, j)$ can be numbered from 1 to $N$; the neighbourhood states of $(i, j)$ can therefore be denoted $\left(s_{1}, s_{2}, \ldots, s_{N}\right)$. Two common neighbourhoods are the five-cell von Neumann neighbourhood $\{(0,0),( \pm 1,0),(0, \pm 1)\}$ and the nine-cell Moore neighbourhood $\{(0,0),( \pm 1,0),(0, \pm 1),( \pm 1, \pm 1)\}$. Periodic boundary conditions are applied at the edges of the lattice so that complete neighbourhoods exist for every cell in $L$.

- $f$ is the update rule. $f$ computes the state $s_{1}(t+1)$ of a given cell from the states $\left(s_{1}, s_{2}, \ldots, s_{N}\right)$ of cells in its neighbourhood: $s_{1}(t+1)=f\left(s_{1}, s_{2}, \ldots, s_{N}\right)$. A quiescent state $s_{q}$ satisfies $f\left(s_{q}, s_{q}, \ldots, s_{q}\right)=s_{q}$.

The collection of states for all cells in $L$ is known as a configuration $c$. The global rule $F$ maps the whole automaton forward in time; it is the synchronous application of $f$ to each cell. The behaviour of a particular $\mathcal{A}$ is the sequence $c^{0}, c^{1}, c^{2}, \ldots, c^{T-1}$, where $c^{0}$ is the initial configuration (IC) at $t=0$.

CA behaviour is sensitive to the IC and to $L, S, N$ and $f$. The behaviour is generally nonlinear and sometimes very complex; no single mathematical analysis can describe, or even estimate, the behaviour of an arbitrary automaton. The vast size of the rule space, and the fact that this rule space is unstructured, 
mean that knowledge of the behaviour a particular cellular automaton, or even of a set of automata, gives no insight into the behaviour of any other CA. In the lack of any practical model to predict the behaviour of a $\mathrm{CA}$, the only feasible method is to run simulations. Fig. 1 illustrates some experimental configurations generated by the authors to demonstrate the capabilities of CA in exhibiting complex behaviour with visually pleasing qualities.
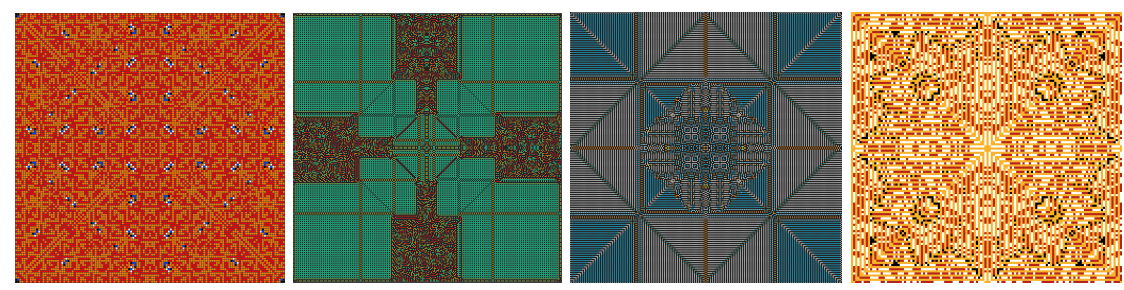

Fig. 1. Samples of multi-state 2D CA patterns

\section{Spatial Complexity Measure of 2D Patterns}

The introduction of information theory by Shannon provided a mathematical model to measure the order and complexity of systems. Shannon's information theory was an attempt to address communication over an unreliable channel [12]. Entropy is the core of this theory [4]. Let $\mathcal{X}$ be discrete alphabet, $X$ a discrete random variable, $x \in \mathcal{X}$ a particular value of $X$ and $P(x)$ the probability of $x$. Then the entropy, $H(X)$, is:

$$
H(X)=-\sum_{x \in \mathcal{X}} P(x) \log _{2} P(x)
$$

The quantity $H$ is the average uncertainty in bits, $\log _{2}\left(\frac{1}{p}\right)$ associated with $X$. Entropy can also be interpreted as the average amount of information needed to describe $X$. The value of entropy is always non-negative and reaches its maximum for the uniform distribution, $\log _{2}(|\mathcal{X}|)$ :

$$
0 \leqslant H \leqslant \log _{2}(|\mathcal{X}|)
$$

The lower bound of relation (2) corresponds to a deterministic variable (no uncertainty) and the upper bound corresponds to a maximum uncertainty associated with a random variable. Another interpretation of entropy is as a measure of order and complexity. A low entropy implies low uncertainty so the message is highly predictable, ordered and less complex. And high entropy implies a high uncertainty, less predictability, highly disordered and complex. Despite the dominance of Shannon entropy as a measure of complexity, it fails to reflect on structural characteristics of $2 \mathrm{D}$ patterns. The main reason for this drawback is 
that it only reflects on the distribution of the symbols, and not on their ordering. This is illustrated in Fig. 2 where, following [1], the entropy of 2D patterns with various structural characteristics is evaluated. Fig. 2a-b are patterns with ordered structures and Fig. 2c is a pattern with repeated three element structure over the plane. Fig. $2 \mathrm{~d}$ is a fairly structureless pattern.

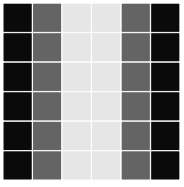

(a)

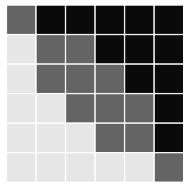

(b)

$$
H=1.5850
$$

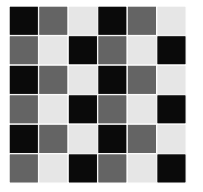

(c)

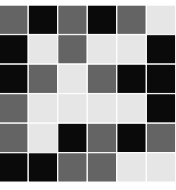

(d)

Fig. 2. Measure of $H$ for structurally different patterns with uniform distribution of elements

Fig. 2 clearly demonstrates the failure of entropy to discriminate structurally different $2 \mathrm{D}$ patterns. In other words, entropy is invariant to spatial rearrangement of composing elements. This is in contrast to our intuitive perception of the complexity of patterns and is problematic for the purpose of measuring the complexity of multi-state 2D CA behaviour.

Taking into account our intuitive perception of complexity and structural characteristics of $2 \mathrm{D}$ patterns, a complexity measure must be bounded by two extreme points of complete order and disorder. It is reasonable to assume that regular structures, irregular structures and structureless patterns lie along between these extremes, as illustrated in Fig. 3.

$$
\text { order } \stackrel{\text { regular structure } \mid \text { irregular structure } \mid \text { structureless }}{\longleftrightarrow} \text { disorder }
$$

Fig. 3. The spectrum of spatial complexity.

A complete regular structure is a pattern of high symmetry, an irregular structure is a pattern with some sort of structure but not as regular as a fully symmetrical pattern and finally a structureless pattern is a random arrangement of elements.

A measure introduced in $[2,13,1]$ and known as information gain, has been suggested as a means of characterising the complexity of dynamical systems and of images. It measures the amount of information gained in bits when specifying the value, $x$, of a random variable $X$ given knowledge of the value, $y$, of another random variable $Y$,

$$
G_{x, y}=-\log _{2} P(x \mid y) .
$$


$P(x \mid y)$ is the conditional probability of a state $x$ conditioned on the state $y$. Then the mean information gain, $\bar{G}_{X, Y}$, is the average amount of information gain from the description of the all possible states of $Y$ :

$$
\bar{G}_{X, Y}=\sum_{x, y} P(x, y) G_{x, y}=-\sum_{x, x} P(x, y) \log _{2} P(x \mid y)
$$

where $P(x, y)$ is the joint probability, $\operatorname{prob}(X=x, Y=y) \cdot \bar{G}$ is also known as the conditional entropy, $H(X \mid Y)[4]$. Conditional entropy is the reduction in uncertainty of the joint distribution of $X$ and $Y$ given knowledge of $Y, H(X \mid Y)=$ $H(X, Y)-H(Y)$. The lower and upper bounds of $\bar{G}_{X, Y}$ are

$$
0 \leqslant \bar{G}_{X, Y} \leqslant \log _{2}|\mathcal{X}|
$$

where $y \in \mathcal{Y}$.

In principle, $\bar{G}$ can be calculated for a $2 \mathrm{D}$ pattern by considering the distribution of cell states over pairs of cells $r, s$,

$$
\bar{G}_{r, s}=-\sum_{s_{r}, s_{s}} P\left(s_{r}, s_{s}\right) \log _{2} P\left(s_{r}, s_{s}\right)
$$

where $s_{r}, s_{s}$ are the states at $r$ and $s$. Since $|\mathcal{S}|=N, \bar{G}_{r, s}$ is a value in $[0, N]$.

In particular, horizontal and vertical near neighbour pairs provide four MIGs, $\bar{G}_{(i, j),(i+1, j)}, \bar{G}_{(i, j),(i-1, j)}, \bar{G}_{(i, j),(i, j+1)}$ and $\bar{G}_{(i, j),(i, j-1)}$. In the interests of notational economy, we write $\bar{G}_{s}$ in place of $\bar{G}_{r, s}$, and omit parentheses, so that, for example, $G_{i+1, j} \equiv G_{(i, j),(i+1, j)}$. The relative positions for non-edge cells are given by matrix $M$ :

$$
M=\left[\begin{array}{cc} 
& (i, j+1) \\
(i-1, j) & (i, j) \\
& (i, j-1)
\end{array}\right] .
$$

Correlations between cells on opposing lattice edges are not considered. Fig. 4 provides an example. The depicted pattern is composed of four different symbols $S=\{$ light-grey,grey,white,black $\}$. The light-grey cell correlates with two neighbouring white cells $(i+1, j)$ and $(i, j-1)$. On the other hand, The grey cell has four neighbouring cells of which three are white and one is black. The result of this edge condition is that $G_{i+1, j}$ is not necessarily equal to $\bar{G}_{i-1, j}$. Differences between the horizontal (vertical) mean information rates reveal left/right (up/down) orientation.

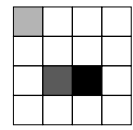

Fig. 4. A sample 2D pattern 
The mean information gains of the sample patterns in Fig. 2 are presented in Fig. 5. The merits of $\bar{G}$ in discriminating structurally different patterns ranging from the structured and symmetrical (Fig. 5a-b), to the partially structured (Fig. 5c) and the structureless and random (Fig. 5d), are clearly evident. The cells in the columns of pattern (a) are completely correlated. However knowledge of cell state does not provide complete predictability in the horizontal direction and, as a consequence, the horizontal $\bar{G}$ is finite. Pattern (b) has non-zero, and identical $\bar{G}$ 's indicating a symmetry between horizontal and vertical directions, and a lack of complete predictability. Analysis of pattern (c) is similar to (a) except the roles of horizontal and vertical directions are interchanged. The four $\bar{G}$ s in the final pattern are all different, indicating a lack of vertical and horizontal symmetry; the higher values show the increased randomness. Details of calculations for a sample pattern are provided in the appendix.

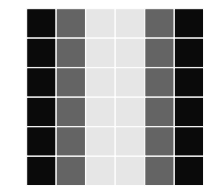

(a) $H=1.5850$

$\bar{G}_{i, j+1}=0$

$\bar{G}_{i, j-1}=0$

$\bar{G}_{i-1, j}=0.8000$

$\bar{G}_{i+1, j}=0.8000 \quad \bar{G}_{i+1, j}=0.7564$

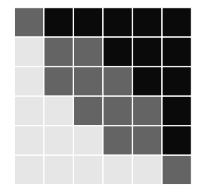

(b)

$H=1.5850$

$\bar{G}_{i, j+1}=0.7564$

$\bar{G}_{i, j-1}=0.7564$

$\bar{G}_{i-1, j}=0.7564$

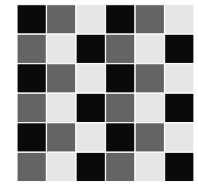

(c)

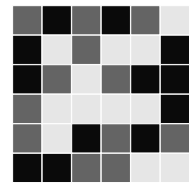

(d)

Fig. 5. The comparison of $H$ with measures of $\bar{G}_{i, j}$ for structurally different patterns.

\section{Experiments and Results}

A set of experiments was designed to examine the effectiveness of $\bar{G}$ in discriminating the particular patterns that are generated by a multi-state $2 \mathrm{D}$ cellular automaton. The (outer-totalistic) CA is specified in Table 1. The chosen experimental rule maps three states, represented by green, red and white; the quiescent state is white.

The experiments are conducted with two different ICs: (1) all white cells except for a single red cell and (2) a random configuration with $50 \%$ white quiescent states (8320 cells), $25 \%$ red and $25 \%$ green. The experimental rule has been iterated synchronously for 150 successive time steps. Fig. 6 and Fig. 7 illustrate the space-time diagrams for a sample of time steps starting from single and random ICs.

The behaviour of cellular automaton from the single cell IC is a sequence of symmetrical patterns (Fig. 6). The directional measurements of $\bar{G}_{i, j}$ for the 
Table 1. Specifications of experimental cellular automaton

$L=129 \times 129$ (16641 cells).

$S=\{0,1,2\} \equiv\{$ white, red, green $\}$

$\mathcal{N}$ : von Neumann neighbourhood

$f: S^{9} \mapsto S$

$f\left(s_{i, j}\right)(t)=s_{i, j}(t+1)=\left\{\begin{array}{l}1 \text { if } s_{(i, j)}(t)=1,2 \text { and } \sigma=0-2 \\ 2 \text { if } s_{(i, j)}(t)=2,3 \text { and } \sigma=1 \\ 2 \text { if } s_{(i, j)}(t)=2 \quad \text { and } \sigma=2 \\ 0 \text { otherwise }\end{array}\right\}$

where $\sigma$ is the sum total of the neighbourhood states.
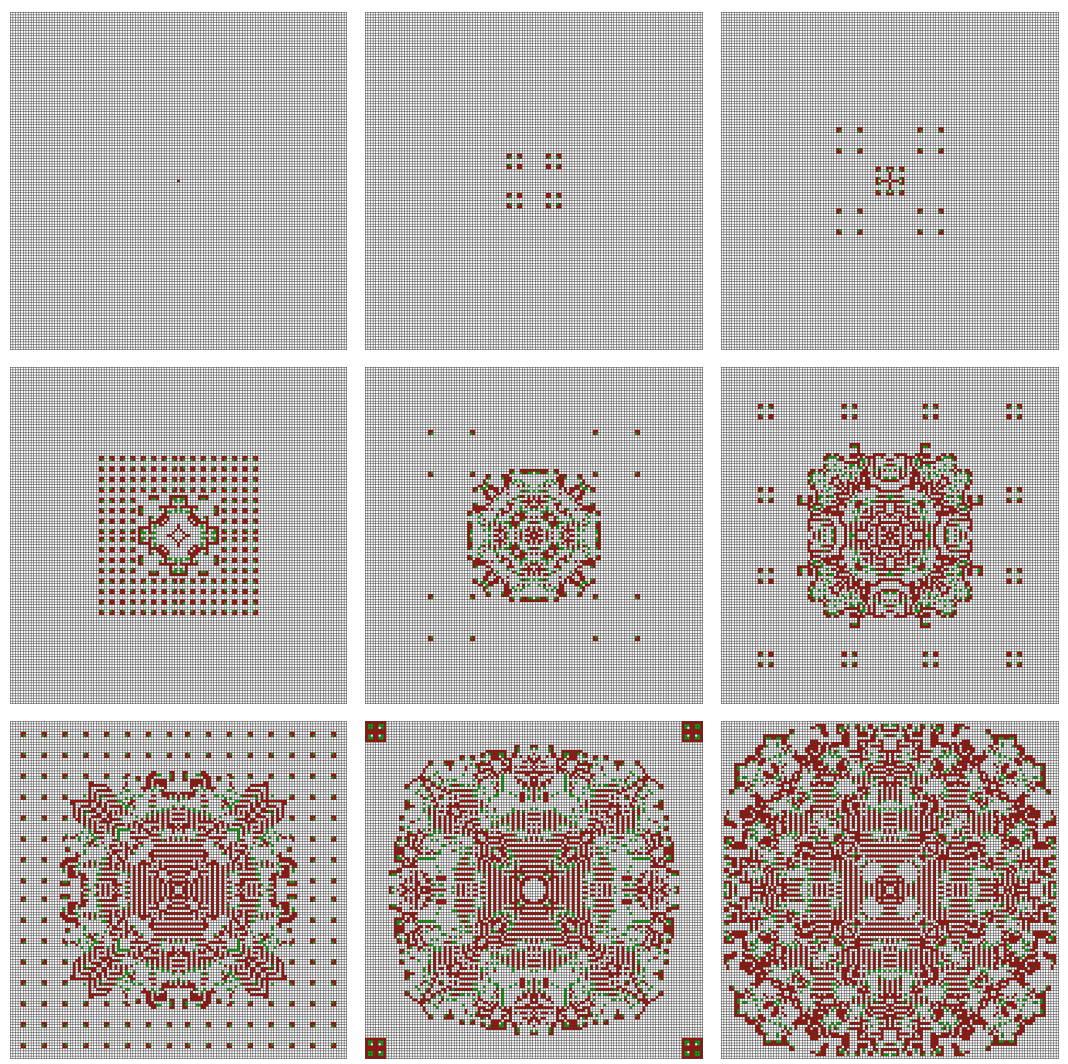

Fig. 6. Space-time diagram of the experimental cellular automaton for sample time steps starting from the single cell IC. 

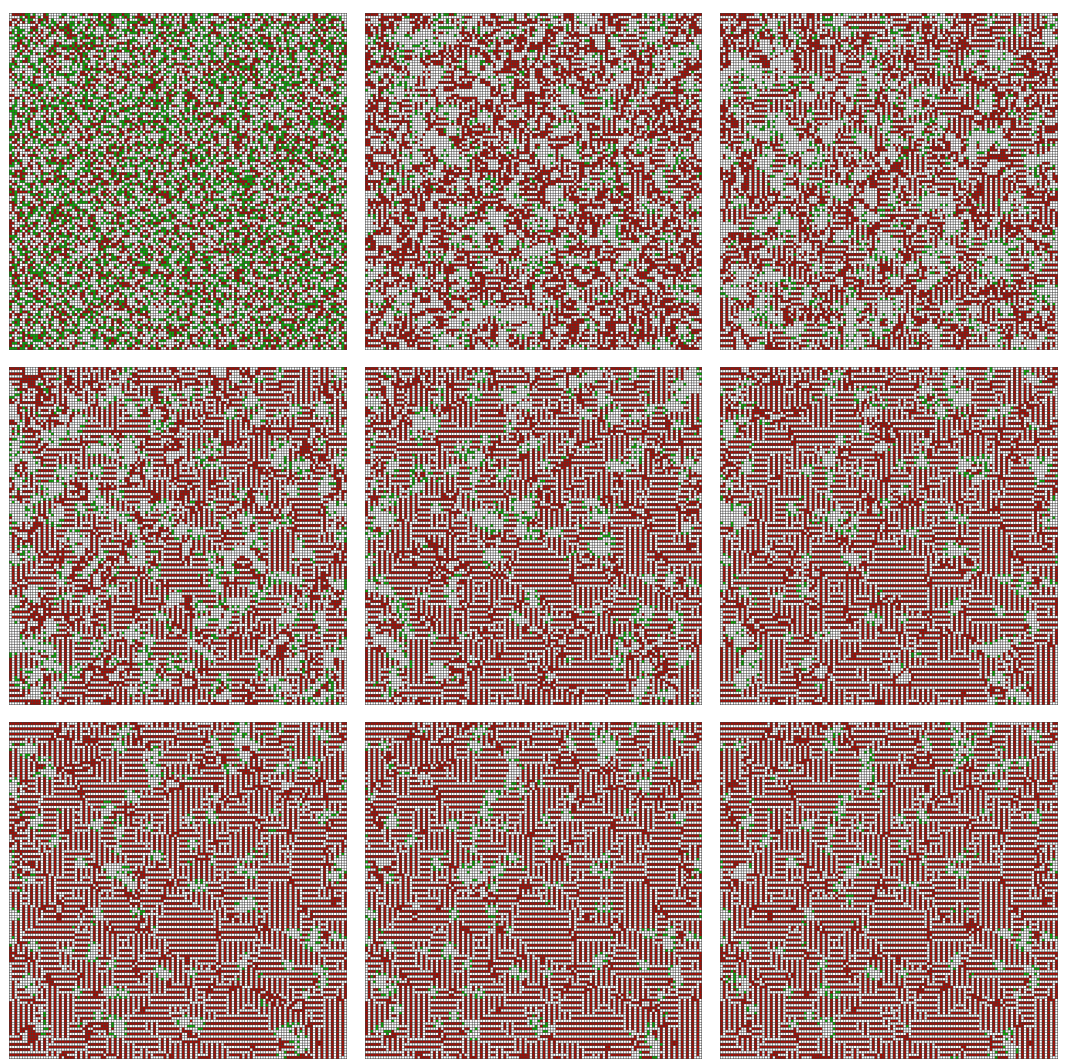

Fig. 7. Space-time diagram of the experimental cellular automaton for sample time steps starting from the random IC.

single cell IC start with $\bar{G}_{i, j+1}=\bar{G}_{i, j-1}=\bar{G}_{i-1, j}=\bar{G}_{i+1, j}=0.00094$ and $H=0.00093$, and they attain $\bar{G}_{i, j+1}=\bar{G}_{i, j-1}=\bar{G}_{i-1, j}=\bar{G}_{i+1, j}=1.13110$ and $H=1.13714$ (and Figs 8,11) at the end of the runs.

The sequence of states can be analysed by considering the differences between the up/down and left/right mean information gains, as defined by

$$
\begin{aligned}
\Delta \bar{G}_{i, j \pm 1} & =\left|\bar{G}_{i, j+1}-\bar{G}_{i, j-1}\right| \\
\Delta \bar{G}_{i \pm 1, j} & =\left|\bar{G}_{i+1, j}-\bar{G}_{i-1, j}\right| .
\end{aligned}
$$

For the single cell IC, $\Delta \bar{G}_{i, j \pm 1}$ and $\Delta \bar{G}_{i \pm 1, j}$ are constant for the 150 time steps $\left(\Delta \bar{G}_{i, j \pm 1}=\Delta \bar{G}_{i, j \pm 1}=0\right)$. This indicates the development of the symmetrical patterns along the up/down and left/right directions.

The behaviour of cellular automaton from the random IC is a sequence of irregular structures (Fig. 7). The formation of patterns with local structures has reduced the values of $\bar{G}_{i, j}$ until a stable oscillating pattern is attained (Figs 7, 
9). This is an indicator of the development of irregular structures. However the patterns are not random patterns since $\bar{G}_{i, j} \approx 1.1$ is less than the maximum three-state value $\log _{2}(3)=1.5850$ (see Eq. 5). Mean information rate differences $\Delta \bar{G}_{i, j \pm 1}$ and $\Delta \bar{G}_{i \pm 1, j}$ for both ICs are plotted in Fig. 10. The structured but asymmetrical patterns emerging from the random start are clearly distinguished from the symmetrical patterns of the single cell IC.

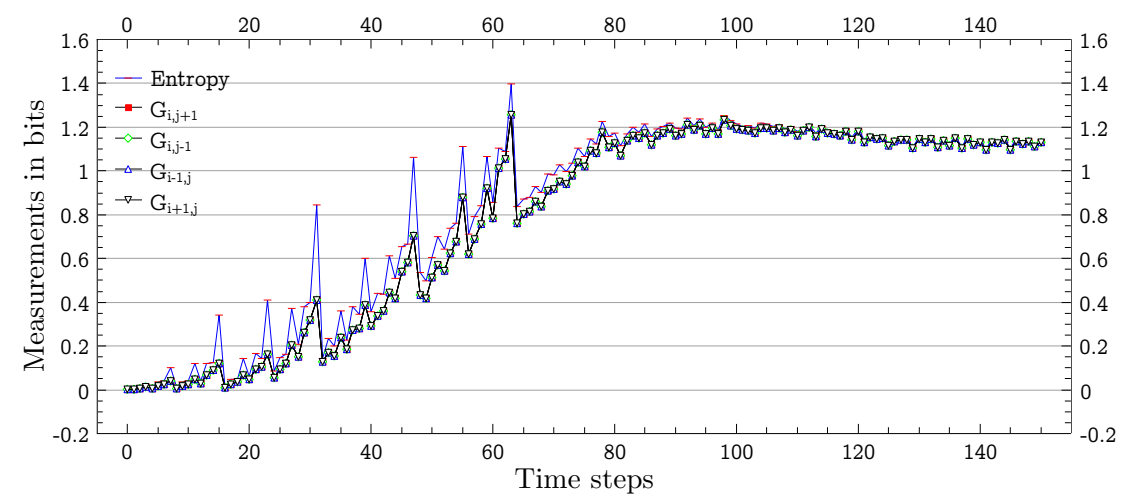

Fig. 8. Measurements of $H, \bar{G}_{i, j+1}, \bar{G}_{i, j-1}, \bar{G}_{i+1, j}, \bar{G}_{i-1, j}$ for 150 time steps starting from the single cell IC.

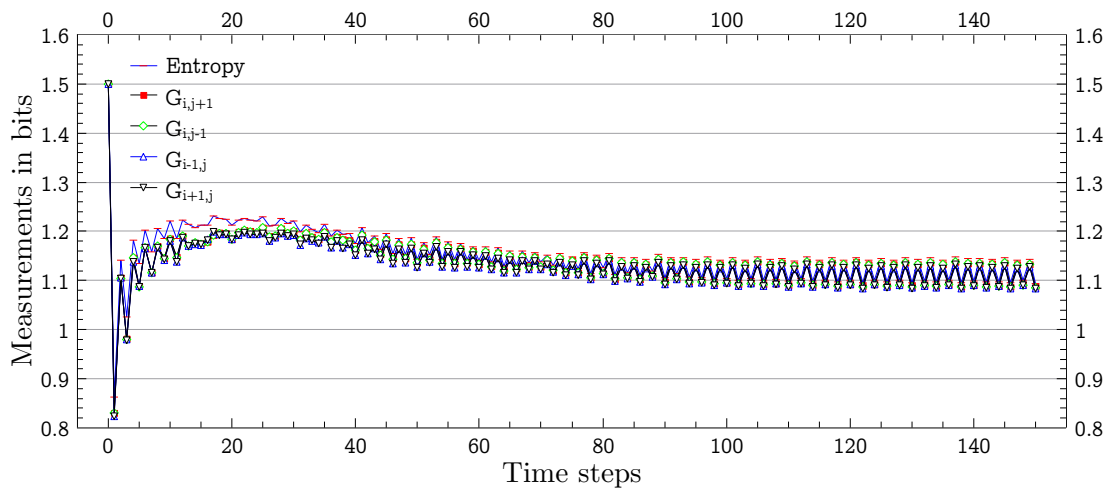

Fig. 9. Measurements of $H, \bar{G}_{i, j+1}, \bar{G}_{i, j-1}, \bar{G}_{i+1, j}, \bar{G}_{i-1, j}$ for 150 time steps starting from the random IC.

These experiments demonstrate that a cellular automaton rule seeded with different ICs leads to the formation of patterns with structurally different characteristics. The gradient of the mean information rate along lattice axes is able to detect the structural characteristics of patterns generated by this particular 


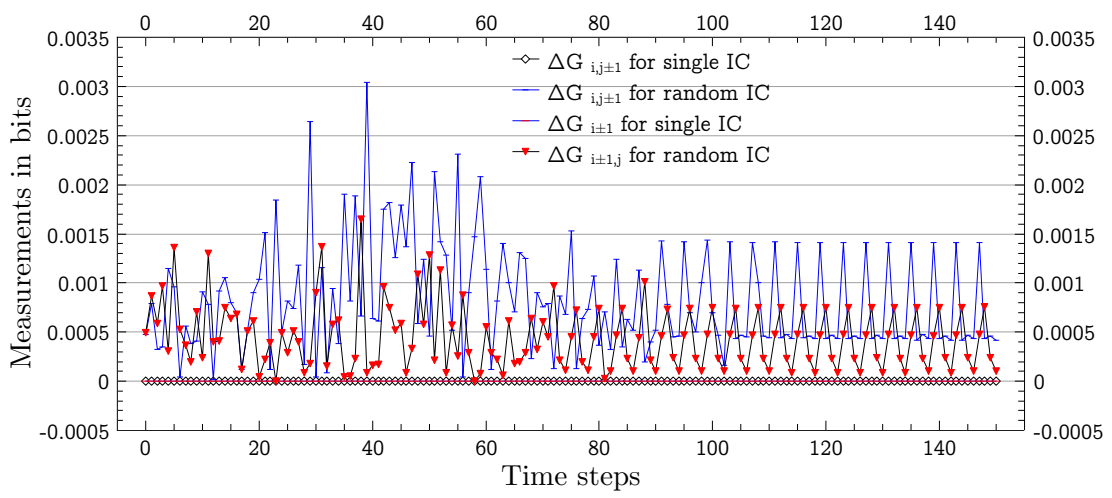

Fig. 10. Plots of $\Delta \bar{G}_{i, j \pm 1}$ and $\Delta \bar{G}_{i \pm 1, j}$ for two different ICs

multi-state 2D CA. From the comparison of $H$ with $\Delta \bar{G}_{i, j \pm 1}$ and $\Delta \bar{G}_{i \pm 1, j}$ in the set of experiments, it is clear that entropy fails to discriminate between the diversity of patterns that can be generated by various CA.

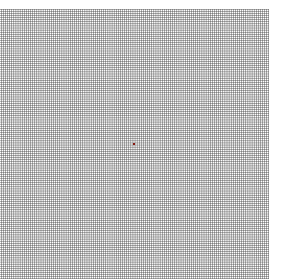

(a)

$t=0$

$H=0.00093$

$\bar{G}_{i, j+1}=0.00094$

$\bar{G}_{i, j-1}=0.00094$

$\Delta \bar{G}_{i, j \pm 1}=0$

$\bar{G}_{i-1, j}=0.00094$

$\bar{G}_{i+1, j}=0.00094$

$\Delta \bar{G}_{i \pm 1, j}=0$

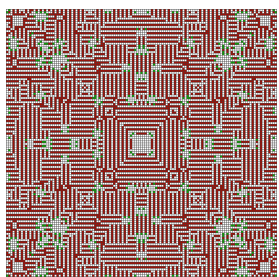

(b)

$t=150$

$H=1.13714$

$\bar{G}_{i, j+1}=1.13110$

$\bar{G}_{i, j-1}=1.13110$

$\Delta \bar{G}_{i, j \pm 1}=0$

$\bar{G}_{i-1, j}=1.13110$

$\bar{G}_{i+1, j}=1.13110$

$\Delta \bar{G}_{i \pm 1, j}=0$

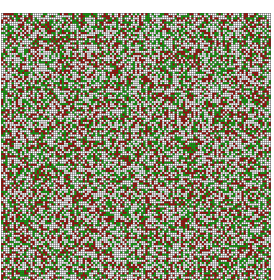

(c)

$t=0$

$H=1.50002$

$\bar{G}_{i, j+1}=1.49924$

$\bar{G}_{i, j-1}=1.49972$

$\Delta \bar{G}_{i, j \pm 1}=0.00048 \quad \Delta \bar{G}_{i, j \pm 1}=0.00042$

$\bar{G}_{i-1, j}=1.50023 \quad \bar{G}_{i-1, j}=1.08318$

$\bar{G}_{i+1, j}=1.49974 \quad \bar{G}_{i+1, j}=1.08308$

$\Delta \bar{G}_{i \pm 1, j}=0.00049 \quad \Delta \bar{G}_{i \pm 1, j}=0.00010$

Fig. 11. Comparison of the cellular automaton's $H$ with four directional measure of $\bar{G}_{i, j} \Delta \bar{G}_{i, j \pm 1}$ and $\Delta \bar{G}_{i \pm 1, j}$ starting from single (a, b) and random ICs (c, d).

\section{Conclusion}

Cellular automata (CA) are one of the early bio-inspired models of self-replicating systems and, in 2D, are powerful tools for the pattern generation. Indeed, multistate $2 \mathrm{D}$ CA can generate many interesting and complex patterns with various 
structural characteristics. This paper considers an information-theoretic classification of these patterns.

Entropy, which is a statistical measure of the distribution of cell states, is not in general able to distinguish these patterns. However mean information gain, as proposed in $[2,13,1]$, takes into account conditional and joint probabilities between pairs of cells and, since it is based on correlations between cells, holds promise for pattern classification.

This paper reports on a pair of experiments for two different initial conditions of an outer-totalistic CA. The potential of mean information gain for distinguishing multi-state 2D CA patterns is demonstrated. Indeed, the measure appears to be particular good at distinguishing symmetry from non-random non-asymmetric patterns.

Since CA are one of the generative tools in computer art, means of evaluating the aesthetic qualities of CA generated patterns could have a substantial contribution towards further automation of CA art. This is the subject of on-going research.

\section{Appendix}

In this example the pattern is composed of two different cells $S=\{$ white, black $\}$ where the set of permutations with repetition is $\{w w, w b, b b, b w\}$. Considering the mean information gain (Eq. 4) and given the positional matrix $M$ (Eq. 7), the calculations can be performed as follows:

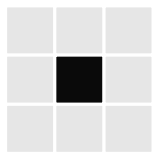

$$
\begin{aligned}
& \text { white - white black-black } \\
& P\left(w, s_{(i, j+1)}\right)=\frac{5}{6} \quad P\left(b, s_{(i, j+1)}\right)=\frac{1}{6} \\
& P\left(w \mid w_{(i, j+1)}\right)=\frac{4}{5} \quad P\left(b \mid b_{(i, j+1)}\right)=\frac{1}{1} \\
& P\left(w, w_{(i, j+1)}\right)=\frac{5}{6} \times \frac{4}{5}=\frac{2}{3} \quad P\left(b, b_{(i, j+1)}\right)=\frac{1}{6} \times \frac{1}{1}=\frac{1}{6} \\
& G\left(w, w_{(i, j+1)}\right)=\frac{2}{3} \log _{2} P\left(\frac{4}{5}\right) \quad G\left(b, b_{(i, j+1)}\right)=\frac{1}{6} \log _{2} P(1) \\
& G\left(w, w_{(i, j+1)}\right)=0.2146 \text { bits } \quad G\left(b, b_{(i, j+1)}\right)=0 \text { bits } \\
& \text { white - black black - white } \\
& P\left(w, s_{(i, j+1)}\right)=\frac{5}{6} \quad P\left(b, s_{(i, j+1)}\right)=\frac{1}{6} \\
& P\left(w \mid b_{(j+1)}\right)=\frac{1}{5} \quad P\left(b \mid w_{(i, j+1)}\right)=\frac{0}{1} \\
& P\left(w, b_{(i, j+1)}\right)=\frac{5}{6} \times \frac{1}{5}=\frac{1}{6} \quad P\left(b, w_{(i, j+1)}\right)=\frac{1}{6} \times 0 \\
& \bar{G}\left(w, b_{(i, j+1)}\right)=\frac{1}{6} \log _{2} P \frac{1}{5} \quad G\left(b, w_{(i, j+1)}\right)=0 \text { bits } \\
& \bar{G}\left(w, b_{(i, j+1)}\right)=0.3869 \text { bits } \\
& \bar{G}=G\left(w, w_{(i, j+1)}\right)+G\left(w, b_{(i, j+1)}\right)+G\left(b, b_{(i, j+1)}\right)+G\left(b, w_{(i, j+1)}\right) \\
& \bar{G}=0.6016 \text { bits }
\end{aligned}
$$

In white - white case $G$ measures the uniformity and spatial property where $P\left(w, s_{(i, j+1)}\right)$ is the joint probability that a cell is white and it has a neighbouring 
cell at its $(i, j+1)$ position, $P\left(w \mid w_{(i, j+1)}\right)$ is the conditional probability of a cell is white given that it has white neighbouring cell at its $(i, j+1)$ position, $P\left(w, w_{(i, j+1)}\right)$ is the joint probability that a cell is white and it has neighbouring cell at its $(i, j+1)$ position, $G\left(w, w_{(i, j+1)}\right)$ is information gain in bits from specifying a white cell where it has a white neighbouring cell at its $(i, j+1)$

position. The same calculations are performed for the rest of cases; black-black, white-black and black-white.

\section{References}

1. Andrienko, Yu. A., Brilliantov, N. V., Kurths, J.: Complexity of two-dimensional patterns. Eur. Phys. J. B 15(3), 539-546 (2000)

2. Bates, J.E., Shepard, H.K.: Measuring complexity using information fluctuation. Physics Letters A 172(6), 416-425 (1993)

3. Brown, P.: Stepping stones in the mist. In: Creative evolutionary systems. pp. 387-407. Morgan Kaufmann Publishers Inc. (2001)

4. Cover, T.M., Thomas, J.A.: Elements of Information Theory (Wiley Series in Telecommunications and Signal Processing). Wiley-Interscience (2006)

5. Frazer, J.: An evolutionary architecture. Architectural Association Publications, Themes VII (1995)

6. Javaheri Javid, M.A., al Rifaie, M.M., Zimmer, R.: Detecting Symmetry in Cellular Automata Generated Patterns Using Swarm Intelligence. In: Dediu, A.H., Lozano, M., Martín-Vide, C. (eds.) Theory and Practice of Natural Computing. Lecture Notes in Computer Science, vol. 8890, pp. 83-94. Springer International Publishing (2014)

7. Javaheri Javid, M.A., te Boekhorst, R.: Cell Dormancy in Cellular Automata. In: Alexandrov, V.N., van Albada, G.D., Sloot, P.M.A., Dongarra, J. (eds.) International Conference on Computational Science (3). Lecture Notes in Computer Science, vol. 3993, pp. 367-374. Springer (2006)

8. Langton, C.G.: Studying artificial life with cellular automata. Physica D: Nonlinear Phenomena 22(1), 120-149 (1986)

9. Miranda, E.: Composing Music with Computers. No. v. 1 in Composing Music with Computers, Focal Press (2001)

10. Scha, I.R.: Kunstmatige Kunst. De Commectie 2(1), 4-7 (2006)

11. Schwartz, L., Schwartz, L.: The Computer Artist's Handbook: Concepts, Techniques, and Applications. W W Norton \& Company Incorporated (1992)

12. Shannon, C.: A mathematical theory of communication. The Bell System Technical Journal 27, 379-423 \& 623-656 (Oct 1948)

13. Wackerbauer, R., Witt, A., Atmanspacher, H., Kurths, J., Scheingraber, H.: A comparative classification of complexity measures. Chaos, Solitons \& Fractals 4(1), 133-173 (1994)

14. Wolfram, S.: Statistical mechanics of cellular automata. Reviews of modern physics 55(3), 601-644 (1983)

15. Wolfram, S.: Universality and complexity in cellular automata. Physica D: Nonlinear Phenomena 10(1), 1-35 (1984)

16. Wolfram, S.: A New Kind of Science. Wolfram Media Inc. (2002)

17. Xenakis, I.: Formalized music: thought and mathematics in composition. Pendragon Press (1992) 